\title{
Lowe Syndrome: A Complex Clinical Diagnosis with a Novel Mutation in the OCRL Gene
}

\author{
Akanksha C. Parikh ${ }^{1}$ Pradnya Gadgil ${ }^{1}$ \\ ${ }^{1}$ Kokilaben Dhirubhai Ambani Hospital and Medical Research \\ Institute, Mumbai, Maharashtra, India \\ J Child Sci 2021;11:e45-e48.
}

\begin{abstract}
Address for correspondence Akanksha Parikh, MBBS, MD, DNB Paediatrics, 7, Milan, Dixit Road, Vile Parle (East), Mumbai 400057, Maharashtra, India (e-mail: gandhi.akanksha@gmail.com).
\end{abstract}

\begin{abstract}
Keywords

- oculocerebrorenal syndrome

- rickets

- growth hormone deficiency

- nonsense mutation

Lowe syndrome (LS) is a rare X-linked condition having a clinical triad of congenital cataracts, intellectual disability, and progressive tubular nephropathy. Although the easily recognizable symptom complex usually evolves by infancy, a unifying diagnosis is often missed. We present a young boy with a prolonged history of multisystem affection, finally leading to the clinical suspicion of LS. The diagnosis was confirmed on genetic analysis as well as a previously unreported mutation in the OCRL gene was discovered. A 9-year-old boy with intellectual disability and recent onset seizures was referred for the evaluation of rickets. In addition, there was a significant past history of neonatal cataracts, infantile glaucoma, persistent albuminuria, and severe short stature with growth hormone deficiency. The characteristic involvement of eyes, brain, and kidneys along with a family history of a maternal uncle being similarly affected led to the clinical suspicion of LS. A whole exome sequencing was performed, which not only confirmed a nonsense mutation, c.2530C $>T$, in exon 23 of the Lowe gene (OCRL) but also revealed it to be a novel pathogenic variant. This case highlights the importance of piecing together the different facets of a complex clinical syndrome in reaching a challenging diagnosis. Also, LS must be kept as a differential in any child with neonatal cataracts and intellectual disability. Genetic confirmation of LS in our patient partly relieved the parental anxiety, and the child continued to remain under followup with multiple specialists, only now with a definite diagnosis.
\end{abstract}

\section{Introduction}

Lowe syndrome (LS) or oculocerebrorenal syndrome is a rare $\mathrm{X}$-linked recessive disorder predominantly involving the eyes, brain, and kidneys. Its prevalence is estimated to be 1:500,000 in the western population. ${ }^{1}$ The gene responsible for LS (OCRL) is situated on Xq25-26 and encodes the ubiquitous enzyme inositol polyphosphate 5-phosphatase. ${ }^{2}$ Mutation in the Lowe gene entails intracellular accumulation of the OCRL protein causing disruption of endocytic trafficking, cell adhesion, and cell migration. ${ }^{3}$ This may explain the progressive multisystem affection seen in LS.

The clinical diagnosis of $\mathrm{LS}$ relies on the presence of an easily recognizable but often missed symptom complex. The concurrent presence of congenital cataracts (100\%), glaucoma (50\%), intellectual disability (75\%), seizures (50\%), behavior prob- lems, and low-molecular-weight proteinuria (100\%) with or without Fanconi syndrome leads one to the clinical suspicion of LS. ${ }^{1,4,5}$ Severe postnatal growth failure is a well-established feature of LS, with renal rickets and cryptorchidism being the other endocrinological manifestations described. ${ }^{6}$

We present a young boy with a prolonged clinical trajectory from one systemic affection to another finally leading to the clinical suspicion of LS. The diagnosis was not only confirmed with genetic analysis but also a novel mutation in the OCRL gene was discovered.

\section{Case Presentation}

A 9-year-old boy with profound intellectual disability and recent onset seizures was referred for evaluation of rickets. There was no history of tetany, polyuria, polydipsia, received

November 27, 2020 accepted after revision January 18,2021
DOI https://doi.org/

10.1055/s-0041-1724042. ISSN 2474-5871.

\footnotetext{
(C) 2021. The Author(s).

This is an open access article published by Thieme under the terms of the Creative Commons Attribution License, permitting unrestricted use, distribution, and reproduction so long as the original work is properly cited. (https://creativecommons.org/licenses/by/4.0/)

Georg Thieme Verlag KG, Rüdigerstraße 14, 70469 Stuttgart, Germany
} 
fractures, or limb deformities. In the past, the child had been diagnosed and adequately treated for vitamin D deficiency rickets at 5 years of age but was not continued on cholecalciferol maintenance therapy thereafter.

The child required frequent medical visits since birth. He was born of a nonconsanguineous union, delivered at term gestation, and had a birth weight of $2.5 \mathrm{~kg}$. The antenatal period and the immediate postnatal period were uneventful. There was a past history of neonatal bilateral dense cataracts and left eye glaucoma requiring surgical correction during infancy for the same. Additionally, he had global delay of milestones, epilepsy, as well as behavioral problems in the form of hyperactivity and temper tantrums. At the age of 8 years, the child was evaluated for profound short stature. A low insulin-like growth factor-1 level of $3.23 \mathrm{nmol} / \mathrm{L}$ (normal range 10.6-33.4 nmol/L) and a peak stimulated growth hormone response of $2.10 \mathrm{ng} / \mathrm{mL}$ confirmed severe growth hormone deficiency. The corresponding bone age was markedly delayed and other pituitary hormones were found to be normal. Recombinant growth hormone ( $\mathrm{rGH}$ ) therapy was initiated at the dose of $0.23 \mathrm{mg} / \mathrm{kg} /$ week. However, stating unsatisfactory response, the parents discontinued the treatment after 5 months of compliant therapy. Past records also revealed persistent albuminuria, without glycosuria in multiple urine routine samples since the neonatal period. A detailed family history revealed a maternal uncle who had similar bilateral cataracts and intellectual disability and had died at 4 years of age due to an undocumented illness. The boy's parents and teenage sister were apparently asymptomatic.

On examination, the child was restless and had poor cognitive abilities. His vital signs were within normal limits. He weighed $20.5 \mathrm{~kg}$ (standard deviation [SD]: -1.94), with a height of $105.8 \mathrm{~cm}$ (SD: -4.61$)$, and had a body mass index of $17 \mathrm{~kg} / \mathrm{m}^{2}$ (SD: +0.72 ). Some facial dysmorphisms with left ocular abnormalities were observed as shown in - Fig. 1. There were no visible signs of rickets except for mild wrist widening. The child was prepubertal without features of hypogonadism. He walked with an unsteady gait and his speech content was severely reduced. He had generalized hypotonia with absent deep tendon reflexes and muscle strength was grade $3+$ in all four limbs. The rest of the systemic examination was unremarkable.

At presentation, the child's biochemical and radiologic investigations confirmed vitamin D deficiency and rickets as shown in - Table 1. Blood and urinary evaluation for renal tubulopathy was performed after treatment of vitamin D deficiency (-Table 1). Following therapy with cholecalciferol, the serum phosphorus and 25-hydroxy vitamin D levels improved, however the alkaline phosphatase remained high and albuminuria persisted. Serum electrolytes were normal and venous blood gas did not reveal obvious acidosis (-Table 1). Hypercalciuria was detected by an increased spot urinary calcium/creatinine ratio and ultrasonography of the kidneys revealed bilateral nephrocalcinosis. The child's past investigations have also been depicted in - Table 1. Cranial neuroimaging revealed dilated perivascular spaces in bilateral periventricular frontal white matter (-Fig. 2) and left optic nerve atrophy (not shown);

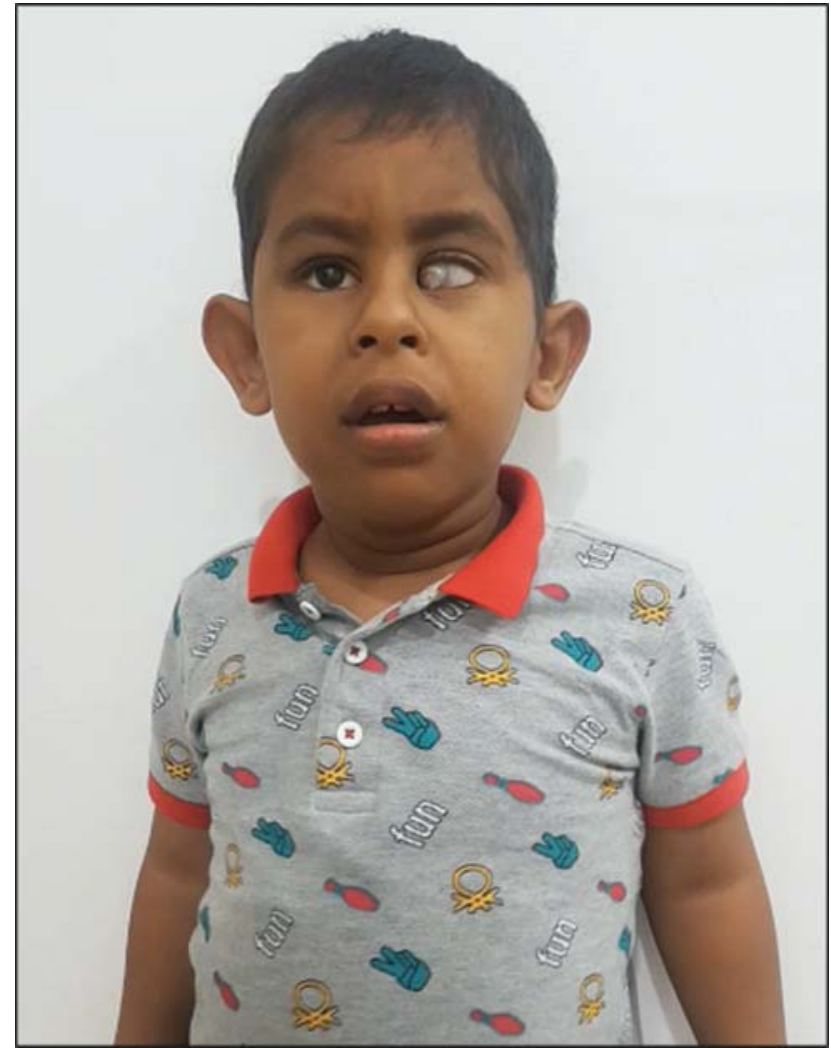

Fig. 1 Clinical photograph revealing left eye corneal scarring and esotropia, and facial dysmorphisms in the form of inverted "v" shaped upper lip, deep set eyes, and low set prominent ears.

pituitary gland was normal in size. In view of the suspicion for LS, a whole exome analysis by next-generation sequencing was requested using a venous blood sample. The sample was processed by an ISO-International organization for Standardization-certified genetic laboratory using Illumina sequencing platform (San Diego, California). Analysis revealed a novel pathogenic mutation, c.2530C $>\mathrm{T}$, in exon 23 of the OCRL gene resulting in the change of amino acid arginine to a premature stop codon (p.Arg844Ter).

In a child with intellectual disability, neonatal cataracts, and nephropathy, a possibility of congenital infection such as rubella was initially considered. However, TORCH titers-serologic tests for TOxoplasma, syphilis, Rubella, Cytomegalovirus, and Herpes simplex-performed in the first week of life were documented to be negative. Zellweger syndrome and congenital myotonic dystrophy are two other conditions presenting with congenital cataracts, intellectual disability, and hypotonia. However, they differ by the presence of distinct facial dysmorphisms and absence of renal tubulopathy. In the present case, the classical oculocerebrorenal involvement with additional features such as glaucoma, growth retardation, and rickets, along with a significant family history, raised the possibility of LS.

In view of vitamin D refractory rickets, the possibility of mild tubular dysfunction was considered, and child was started on a low-dose alkali with $3 \mathrm{mEq} / \mathrm{kg} /$ day of sodium bicarbonate. Cholecalciferol was continued in monthly maintenance doses of 60,000 IU. Likewise, the treatment of other systemic involvement was mainly supportive. 
Table 1 Past and present investigations

\begin{tabular}{|l|l|l|l|l|}
\hline Blood investigations & 4 years & 4 years $^{\mathrm{a}}$ & $\mathbf{9}$ years & 9 years $^{\mathbf{a}}$ \\
\hline $\mathrm{Ca}(\mathrm{mg} / \mathrm{dL})$ & 8.8 & 9.2 & 8.6 & 10.1 \\
\hline $\mathrm{P}(\mathrm{mg} / \mathrm{dL})$ & 2.5 & 5.6 & 2.0 & 5.4 \\
\hline $\mathrm{S} . \mathrm{ALP}(\mathrm{IU} / \mathrm{L})$ & 788 & 469 & 609.5 & 658 \\
\hline $25(\mathrm{OH})$ vitD $(\mathrm{ng} / \mathrm{mL})$ & 8.9 & 45.7 & 5.0 & 95.1 \\
\hline $\mathrm{PTH}(\mathrm{pg} / \mathrm{mL})$ & - & - & - & 13.7 \\
\hline $\mathrm{Creat}(\mathrm{mg} / \mathrm{dL})$ & 0.47 & - & 0.37 & - \\
\hline $\mathrm{Na} / \mathrm{K} / \mathrm{Cl}(\mathrm{mEq} / \mathrm{L})$ & $139 / 4.6 / 106$ & - & $139 / 3.7 / 102$ & $141 / 4.5 / 103.9$ \\
\hline $\mathrm{Mg}(\mathrm{mg} / \mathrm{dL})$ & - & - & 2.4 & - \\
\hline $\mathrm{PH} / \mathrm{HCO}_{3}^{-}(\mathrm{mEq} / \mathrm{L})$ & $7.2 / 17.7$ & - & $7.33 / 19.1$ & $7.32 / 23.5$ \\
\hline
\end{tabular}

Abbreviations: $25(\mathrm{OH})$ vitD, 25-hydroxy vitamin D; ALP, alkaline phosphatase; Ca, calcium; Cl, chloride; Creat, $\mathrm{Creatinine} \mathrm{HCO}_{3}{ }^{-}$, bicarbonate; $\mathrm{K}$, potassium; Mg, magnesium; Na, sodium; P, phosphorus; PTH, parathyroid hormone.

${ }^{\text {a After }} 6$ weeks of treatment with weekly oral cholecalciferol $(60,000 \mathrm{IU})$.

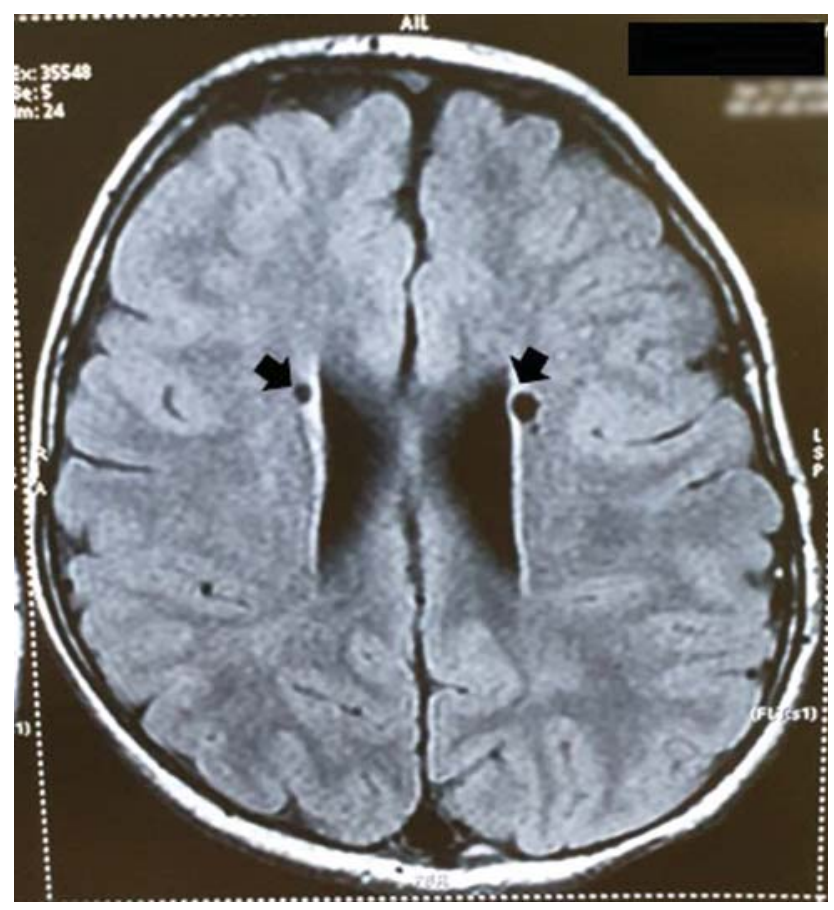

Fig. 2 Axial FLAIR-fluid-attenuated inversion recovery-image shows small cerebrospinal fluid signal intensity cysts in the frontal periventricular regions bilaterally consistent with dilated perivascular spaces (black arrows). Overall myelination is complete for age.

Special schooling, behavior therapy, topical medication for glaucoma, and anticonvulsants were continued as advised by the respective specialists.

The genetic confirmation of diagnosis led to the alleviation of parental anxiety to a certain extent. The child continued to remain under follow-up with multiple specialists, only now with a unifying diagnosis. Importantly, being an X-linked disease, parents were counseled regarding the future risk of disease transmission, and genetic screening of the mother and the elder female sibling was discussed. An ophthalmological examination to look for lenticular changes as a predictor of carrier status revealed lenticular punctate opacities in the mother, whereas the sister showed no lenticular involvement.

\section{Discussion}

Our patient had the classical clinical triad of LS, viz, cataracts, intellectual disability, and renal tubular dysfunction. ${ }^{7}$ The diagnosis was confirmed on genetic analysis during which a novel pathogenic variant in the OCRL gene was discovered.

Central hypotonia with absent deep tendon reflexes, cataracts, and low-molecular-weight proteinuria are universal features of LS, manifesting in the neonatal period. ${ }^{1}$ Subsequently, other features of proximal tubular dysfunction such as phosphaturia, aminoaciduria, hypercalciuria, and renal tubular acidosis may develop, thereby manifesting as failure to thrive in the first few months of life. ${ }^{4}$ Interestingly, glycosuria is absent in a vast majority of patients leading to believe that LS involves a selective tubular dysfunction and not a generalized Fanconi syndrome. ${ }^{8}$ Chronic kidney disease (CKD) eventually ensues, with approximately one-third of children having advanced renal disease at the time of diagnosis. ${ }^{3}$ Serum creatinine may not be a reliable marker of CKD in these patients due to low muscle mass, and instead serum cystatin C may be preferred to estimate the glomerular filtration rate. ${ }^{6}$ Our patient had been diagnosed with vitamin D deficiency for the second time and although his serum phosphorous, vitamin $\mathrm{D}$, and parathyroid hormone levels returned to normal with cholecalciferol treatment, alkaline phosphatase showed no improvement. The vitamin D refractory rickets may be explained by an underlying mild renal tubular dysfunction warranting the initiation of a low-dose alkali.

Children with LS have a normal length at birth with progressive growth failure being apparent after infancy. ${ }^{9}$ Metabolic acidosis, dyselectrolytemia, renal rickets, and CKD all contribute to the growth failure. ${ }^{3}$ As seen in our patient, growth hormone deficiency with marked bone age delay has been previously reported. ${ }^{10,11}$ Although some benefit with rGH therapy has been described irrespective of the growth hormone status in children with LS, additional data are required for any conclusive evidence. ${ }^{12}$ Cryptorchidism is seen in approximately one-third of 
children with LS; however, most children progress through puberty normally. 6,9

Cranial neuroimaging findings in children with LS are usually nonspecific and do not correlate with disease severity. ${ }^{13}$ As seen in the present clinical case, characteristic dilated perivascular space is a commonly described magnetic resonance imaging brain feature in children with LS. ${ }^{14}$ Additionally, T2-weighted white matter hyperintensities may also be observed. ${ }^{15}$ Although the diagnosis is mainly based on clinical and biochemical grounds, such neuroimaging findings can help strengthen the clinical suspicion of LS.

The OCRL gene is composed of 24 exons and encodes the OCRL1 protein, which contains an $N$-terminal domain, a central catalytic phosphatase domain, an ASPM-SPD2-Hydin domain, and an inactive C-terminal Rho-GTPase activating protein (GAP) domain. ${ }^{3}$ Over 170 mutations responsible for LS are known, majority of which include frameshift, nonsense, or splicing defects. ${ }^{5,16}$ The present clinical case had a nonsense mutation in exon 23 , prematurely terminating the 901 amino acid chain of the OCRL protein at the 844th position. The resultant truncated Rho-GAP domain possibly affects the interaction of OCRL1 with clathrin and various cell proteins, hampering endocytosis and cilia formation as well as leading to loss of epithelial cell polarity. ${ }^{3,17}$ The observed variant has not been previously reported in population databases. The in-silico prediction of the mutation had a damaging impact on the OCRL protein function and according to the American College of Medical Genetics guidelines, it was considered as a pathogenic variant. Unfortunately, no genotype-phenotype correlation has been found in LS. Although penetrance is complete, ${ }^{4}$ the same pathological variant has been observed to manifest with varying severity in the same family. ${ }^{3}$ Two-thirds of children with LS are known to occur due to maternal carriers; the presence of punctate lenticular opacities on slit-lamp examination is a noninvasive and inexpensive method to predict carrier status in postpubertal related females, as was performed in the child's mother and sibling. ${ }^{6}$ Ophthalmological examination revealed that the mother is potentially a carrier; however, absence of lenticular opacities in the daughter meant she was likely spared.

Further research is required to address certain unanswered questions regarding LS, such as whether growth hormone deficiency is related to specific OCRL mutations or is a part of the disease pathology itself. Also, the benefit of growth hormone therapy in children with LS and the reason for variability of disease severity with the same mutation remain unknown.
Conflict of Interest

None declared.

\section{References}

1 Loi M. Lowe syndrome. Orphanet J Rare Dis 2006;1(01):16

2 Olivos-Glander IM, Jänne PA, Nussbaum RL. The oculocerebrorenal syndrome gene product is a $105-\mathrm{kD}$ protein localized to the Golgi complex. Am J Hum Genet 1995;57(04):817-823

3 Recker F, Reutter H, Ludwig M. Lowe syndrome/Dent-2 disease: a comprehensive review of known and novel aspects. J Pediatr Genet 2013;2(02):53-68

4 Lewis RA, Nussbaum RL, Brewer EDLowe Syndrome. 2001 Jul 24 [updated 2012 Feb 23]. In: Pagon RA, Bird TD, Dolan CR, Stephens K, Adam MP, eds. GeneReviews ${ }^{\mathrm{TM}}$ [Internet]. Seattle (WA): University of Washington, Seattle1993-2013. Accessed January 31, 2021 at: http://www.ncbi.nlm.nih.gov/books/NBK1480/

5 Bökenkamp A, Levtchenko E, Recker F, Ludwig M. Clinical utility gene card for: Lowe syndrome. Eur J Hum Genet 2015;23(06):889

6 Bökenkamp A, Ludwig M. The oculocerebrorenal syndrome of Lowe: an update. Pediatr Nephrol 2016;31(12):2201-2212

7 Lowe CU, Terrey M, MacLACHLAN EA. Organic-aciduria, decreased renal ammonia production, hydrophthalmos, and mental retardation; a clinical entity. AMA Am J Dis Child 1952;83(02):164-184

8 Recker F, Zaniew M, Böckenhauer D, et al. Characterization of 28 novel patients expands the mutational and phenotypic spectrum of Lowe syndrome. Pediatr Nephrol 2015;30(06):931-943

9 McSpadden K. Living with Lowe Syndrome: A Guide for Families, Friends and Professionals. Chicago, Ridge: Lowe Syndrome Association Inc.; 2010

10 Dai C, Wang L, Li Y, et al. Lowe syndrome with extremely short stature: growth hormone deficiency may be the pathogeny. Growth Factors 2019;37(3-4):170-177

11 Hou JW. Amelioration of hypophosphatemic rickets and osteoporosis with pamidronate and growth hormone in Lowe syndrome. J Formos Med Assoc 2009;108(09):730-735

12 Zaniew M, Bökenkamp A, Kołbuc M, et al. Long-term renal outcome in children with OCRL mutations: retrospective analysis of a large international cohort. Nephrol Dial Transplant 2018;33 (01):85-94

13 de Carvalho-Neto A, Ono SE, de Melo Cardoso G, Santos ML, Celidonio I. Oculocerebrorenal syndrome of Lowe: magnetic resonance imaging findings in the first six years of life. Arq Neuropsiquiatr 2009;67(2A):305-307

14 Demmer LA, Wippold FJ II, Dowton SB. Periventricular white matter cystic lesions in Lowe (oculocerebrorenal) syndrome. A new MR finding. Pediatr Radiol 1992;22(01):76-77

15 Sener RN. Lowe syndrome: proton MR spectroscopy, and diffusion MR imaging. J Neuroradiol 2004;31(03):238-240

16 Hichri H, Rendu J, Monnier N, et al. From Lowe syndrome to Dent disease: correlations between mutations of the OCRL1 gene and clinical and biochemical phenotypes. Hum Mutat 2011;32(04): 379-388

17 Suarez-Artiles L, Perdomo-Ramirez A, Ramos-Trujillo E, ClaverieMartin F. Splicing analysis of exonic OCRL mutations causing Lowe syndrome or dent-2 disease. Genes (Basel) 2018;9(01):15 